\title{
Was wird aus den in den Uterus ejaculierten und nicht zur Befruchtung verwendeten Spermatozoen?
}

\author{
Ven
}

J. Sobotta.

Hierzu Tafel XIV.

Beim Begattungsakt der meisten Säugetiere gelangen bekanntJich sehr große Mengen; meist Millionen von Spermatozoen in die Uterushöhle. Die Masse des ejakulierten Sperma ist bei manchen Säugetieren so enorm groß, daß die Wände des Uterus durch das Ejakulat stark gedehnt werden und das Uterushorn das Mehrfache des Kalibers gegenüber dem Zustand vor der Begattung annimmt. Ich verweise in dieser Hinsich a auf frühere Mitteilungen von mir $(6,7)$.

Von diesen zahllosen im Uterus befindlichen Spermatozoen gelangt nun bloß ein geringer Teil in den Eileiter, worauf ich wohl zuerst ausdrücklich aufmerksam gemacht habe (6). Kürzlich habe ich mich mit dieser Frage nochmals beschäftigt (7) und auch von anderer Seite ist diese Tatsache bestätigt worden. Wenn auch Verschiedenheiten der Zahl der bei verschiedenen Säugetierspezies in die Tuba uterina eindringenden Spermatozoen bestehen, z. B. beim Kaninchen diese Zahl wesentlich größer ist als beim Meerschweinchen und namentlich bei Ratte und Maus, so handelt es sich doch stets nur um eine äußerst geringe Anzahl gegenüber den Millionen von Samenfäden im Uterus. Stets ist es nur ein winziger Teil des Ejakulates, dem die Möglichkeit gegeben wird, seine eigentliche und natürliche Funktion zu erfüllen. Die Hauptmasse aber 
der ejakulierten Spermatozoen verharrt eine Zeitlang zwecklos in den Genitalwegen des weiblichen Tieres, um hier abzusterben.

Entgegen einer ziemlich weit verbreiteten Ansicht, daB dit Spermatozoen im Genitaltraktus des weiblichen Tieres sich längere Zeit lebend erhalten, habe ich den Nachweis führen können (6), $\mathrm{daB}$ sie $z$. B. bei der Maus sehr schnell absterben und ihre Lebensfähigkeit im Uterus dieses Tieres überhaupt nur wenige Stunden beträgt. Das gleiche gilt für die Ratte. Aber auch hierin mögen mancherlei Verschiedenheiten bei den einzelnen Säugetierspezies bestehen, die noch nicht genügend bekannt sind. Bei einzelnen scheint die Lebensdauer der Samenfäden eine wesentlich längere zu sein; so werden bekanntlich die (europäischen) Fledermäuse im Herbst vor Eintritt der kalten Jahreszeit begattet; das Sperma verharrt dann während der ganzen Dauer des Winters in einer Art Kältestarre lebend, bis mit Beginn des Frühjahrs die Befruchtung erfolgt.

Die Frage, was aus den Spermatozoen wird, die im Uterus liegend nicht zur Befruchtung Verwendung finden, und die Frage nach ihrem Schicksal überhaupt ist bisher wenig erörtert worden, wie überhaupt die biologischen Vorgänge bei der Befruchtung von den meisten Untersuchern vernachlässigt worden sind. Erschien ja doch für den Morphologen von Fach das Problem der rein morphologischen Erforschung des Befruchtungsvorgangs das wichtigere und reizvollere und der Physiologe hat weit seltener Gelegenheit, die fraglichen Vorgänge an geeignetem Material zu studieren. Auch waren die von der Mehrzahl der morphologischen Untersucher angewendeten Methoden nicht immer geeignet, die hier zu erörternde Frage zu beantworten.

Daher kommt es, daß eigentlich nur drei Untersucher sich mit dem Schicksal der Spermatozoen im Uterus beschäftigt haben. nämlich außer mir $(6,7)$ nur noch $K$ ö $n$ ig s $t$ e in (2) und $K$ ohlbrugge $(3,4,5)$.

Ich hatte schon vor vielen Jahren kurz auf das Schicksal der Spermatozoen im Uterus der Maus (6) die Aufmerksamkeit gelenkt. Ich machte damals auf Grund sehr zahlreicher Beobachtungen nach Beschreibung des vom Ejakulat prall angefüllten Uterus dieses Tieres folgende Angabe: ,Die Spermatozoen bleiben nur wenige Stunden lebend, liegen aber auch, wenn sie abgestorben sind, noch meist in Klumpen zusammen. Zum Teil werden sie 
durch die Vagina neben dem schon weich gewordenen Vaginalpfropf entleert; zum Teil zerfallen sie wohl schon im Uterus zu körnigem Detritus."

$\mathrm{Zu}$ ganz ähnlichen Resultaten ist $\mathrm{Kön}$ igste in (2) gekommen, der das Schicksal der Spermatozoen sowohl in den männlichen wie in den weiblichen Genitalwegen untersuchte. Für dic letzteren Zwecke benutzte er den Uterus der Ratte nach der Begattung. Er beschreibt èbenfalls die außerordentlich starke Ausdehnung des Organs durch die enorm große Spermamasse (von Streichholz-bis Bleistiftdicke), wobei der Inhalt fast nur aus Spermatozoen besteht. Nach Erweichung des Vaginalpfropfes wird das bisher im Uteruslumen zurückgehaltene Ejakulat größtenteils in die Vagina entleert, also mechanisch entfernt; ein Teil der Spermatozoen bleibt aber im Uterus zurück und wird durch Leukozyten, die in großen Mengen durch das Uterusepithel in die Lichtung einwandern, phagozitiert.

Die gleiche Leukozytose der Uteruswand konnte $\mathrm{K} \ddot{\mathrm{o}} \mathrm{n}$ igs t e in auch bei anderen Säugetieren, z. B. auch bei der Maus feststellen.

Ich selbst habe dann später, aber erst nach der Veröffentlichung von Kohlb rugge $(3,4)$ und ohne die (mir auch nur sehr schwe: zugängliche) kleine Mitteilung von Königstein (l. c.) zu kennen, nochmals mit folgenden Worten zu der Frage Stellung genommen und (7) zwar bei Besprechung des zeitlichen Zusammenhangs zwischen der Erweichung des Vaginalpfropfes und der Abnahme des Volumens des Ejakulates: ,Man wird also wohl annehmen \{ürfen, daß die abgestorbenen Spermatozoen bzw. ihre aufgelösten Reste schließlich per vaginam wieder aus dem Uterus entfernt werden, soweit nicht die Tätigkeit der erst um diese Zeit in nennenswerter Menge im Ejakulat auftretenden Leukozyten eine Rolle bei der Resorption der Spermareste spielen."

Während also die Angaben von Königstein mit den meinigen im wesentlichen übereinstimmen, ist Kohlbrugge $(3,4,5)$ zu ganz anderen Ergebnissen gekommen, gegen die ich seinerzeit (7) schon Stellung nahm. Auch vermutete ich schon damals, wie mehrere der entschieden irrigen Auffassungen von Koh Ibrugge zustande gekommen sein könnten, ich gab vorsichtshalber dieser Vermutung in meiner Polemik keinen Ausdruck. Ich habe aber diese Frage nicht aus dem Auge verloren und so 
führen mich gelegentliche Beobachtungen und die letzte, mit meiner Veröffentlichung (7) zusammen erschienene Mitteilung von $\mathrm{Kohl-}$ b r ugg e (5) wieder auf diesen Gegenstand zurück.

Kohlbrugge (l. c.) nimmt an, daß die Spermatozoen im Uterus der von ihm untersuchten javanischen Fledermaus nicht nur nicht schnell absterben, wie das bei Maus und Ratte sicher, bei vielen anderen Säugetieren wahrscheinlich der Fall ist, sondern daB sie sogar im Uterus noch eine aktive Rolle spielen. Es handelt sich um eine exotische Fledermausart, bei der kein Winterschlaf des Spermas im Uterus stattfindet, wie bei den europäischen. Allerdings wurde die Lebensfähigkeit der Spermatozoen nicht am lebenden Objekt geprüf. Außerdem handelte es sich bei den Spermatozoen, die Kohlbrugge im Uterus der Fledermausspezies beschreibt, vielleicht um solche einer oder mehrerer folgender Begattungen, so daß die Dauer der Lebensfähigkeit der Spermatozoen dieser Spezies auch keine so sehr bedeutende ist.

Wenn ich hier nach 8 Jahren erneut an meine Polemik gegen Koh I brugg e (7) anknüpfe, so ist es dem Leser wohl bequemer, wenn ich den Gegenstand dieser hier nochmals kurz auseinandersetze und daran dann die Besprechung der letzten Publikation von Kohlbrugge (5) arschließe. Kohlbrugge fand, daß die Uteruswand eine Anziehung auf die Spermatozoen ausübt, daB diese massenhaft in die Drüsen eindringen, ja das Uterusepithel durchdringen und sogar ihren Weg bis in die bindegewebigen Teile der Schleimhaut nehmen, daß also direkt eine Invasion der Uteruswand seitens der Spermatozoen erfolgt. Ferner sollen die Spermatozoen auch in die Zellen der Blastula eindringen, wenn diese in den Uterus gelangt ist (dabei handelt es sich wohl sicher um solche einer erneuten Begattung).

Demgegenüber betonte ich, daß von einer Verallgemeinerung der merkwürdigen und bis dahin noch von niemand bestätigten Tätigkeit der Spermatozoen im Uterus der Säugetiere keine Rede sein kann, daß z. B. bei Ratte und Maus die Spermatozoen sehr schnell im Uterus absterben und niemals auch nur die geringste Neigung zeigen, in die Uterusschleimhaut einzudringen, auch spricht keine Tatsache für eine Anziehung der Spermatozoen seitens der Uteruswand. Ebensowenig ist bei Maus und Ratte davon die Rede, daß die Spermatozoen in die Blastula eindringen (7). 
Was wird aus den in den Uterus ejaculierten usw. Spermatozcen? 189

Natürlich ist es ja möglich, daß die Spermatozoen der verschiedenen Säugetiere sich verschieden verhalten. Allerdings finde ich in der ganzen Literatur nur eine einzige teilweise Bestätigung der Befunde von $\mathrm{Kohl}$ b r ug ge, das ist die kürzlich gemachte Notiz von Grosser (1), daB auch beim Maulwurf die Spermatozoen ein ähnliches Verhalten zur Uteruswand zeigen, wie es $\mathrm{Ko}$ h. 1 b r ugg e beschreibt, insofern als sie reihenweise gegen diese mit ihren Köpfen gerichtet sind und auch die Uteruswand betreten können.

Es sind in dieser Hinsicht wohl erst erneute und auf ein umfangreicheres Material basierte Untersuchungen erforderlich, um festzustellen, ob das Betreten der Uteruswand seitens der Spermatozoen ein absolut normales Vorkommnis ist, oder ob es sich nicht etwa um eine agonale Erscheinung der Uterusschleimhaut handelt. Jedenfalls kommt dabei keine allgemein gültige Erscheinung in Frage, und deswegen wandte ich mich seinerzeit (7) gegen die Folgerungen, die Kohlbrugge $(3,4)$ aus seinen Befunden zog.

Ganz besonders aber mußte ich den weiteren Schlußfolgerungen von $\mathrm{Kohlbrugg}$ e widersprechen, daß nämlich die in die Uteruswand eingedrungenen Spermatozoen dort erhebliche Veränderungen erfahren, mit den Zellen der Schleimhaut sich vereinigen usw. sollten (s. unten). Daß in dieser Hinsicht direkte Irrtümer von Kohlbrugge vorliegen müßten, war mir von vornherein klar, auch ahnte ich schon, welcher Art diese sein könnten. Inzwischen ist es mir abei sowohl auf Grund meiner eigenen Befunde als auch des Geständnisses von $\mathrm{Kohlbrugge}$ selbst in seiner letzten Verötfentlichung (5) klar geworden, daß meine Vermutungen richtig seien.

Ich hatte schon in meiner Erwiderung auf die erste Mitteilung von Kohlbrugge (3) auf die ganz unzweckmäBige Fixierung aufmerksam gemacht, die dieser angewandt hatte, und viele der so eigenartigen Behauptungen des Autors aut Verkennung von Kunstprodukten zurückgeführt. Und diese Auffassung hat sich bei mir um so mehr gefestigt, je mehr ich dem Schicksal des Ejakulates im Uterus nachgegangen bin. Kürzlich erhielt ich einige Präparate, die meine Aufmerksamkeit erneut aut diese Frage richteten und den letzten Zweifel an der Auffassung beseitigten, dic ich von Anfang an von den Deutungen der Befunde von Koll l b r g g e hatte. 
Ich gehe zunächst zu meinem eigentlichen Thema über. In meiner letzten diesen Gegenstand behandelnden Mitteilung (7), beschrieb ich das Verhalten des Ejakulates der Maus im Uterus in den verschiedenen, auf die Begattung folgenden Zeitabschnitten und erwähnte dabei, daß, wenn die Spermatozoen ihre Bewegungsfähigkeit eingebüßt haben, ihre Köpfe die sonst so charakteristische Färbbarkeit mit Kernfarbstoffen verlieren und „,daneben Elemente auftreten, die in den Ejakulaten früherer Perioden fehlten, das sind ziemlich zahlreiche Leukozyten“. Die einige Jahre vorher erschienene kleine Mitteilung von Königstein (2) war mir um diese Zeit nicht bekannt.

Ich habe damals die hier zu erörternde Frage nur gestreift, obwohl ich schon im wesentlichen der gleichen Ansicht war, die ich unten entwickeln will und die sich auch mit den Angaben von Königstein in den Hauptpunkten deckt. Ich habe mich noch nicht über die Herkunft dieser Leukozyten geäußert; aber daB sie die Ursache der Hauptirrtümer von Kohlbrugge sein könnten, dieser Verdacht war damals schon in mir aufgetaucht; nur wollte ich ihn ohne erneute positive Unterlagen nicht äußern.

Die Leukozyten, die sich etwa von der achten Stunde post coitum an bei der Maus im Ejakulat des Uterus finden, stammen natürlich aus der Uteruswand. Und gerade deren Betrachtung, die die meisten Untersucher während der fraglichen Zeit vernachlässigt haben, ergibt eine kurze Zeit hindurch ein wirklich überraschendes Bild ihres Baues, das selbst den, der mit ihm gut vertraut ist, einigermaßen $z \mathfrak{u}$ befremden imstande ist.

Um die gleiche Zeit nämlich, in der die Spermatozoen im Uterus absterben, und die ersten Leukozyten in der Uteruslichtung auftreten, also etwa 9 Stunden post coitum, findet eine massenhafte Durchwanderung der Uteruswand seitens farbloser Blutzellen statt.

Während im nichtbrünstigen Zustand des Uterus und in gleicher Weise zur Zeit der Begattung und auch während der übrigen Zeit der Gravidität zwar gelegentlich Leukozyten im Uterusepithel angetroffen werden, ebenso häufig oder selten, wie man diese Erscheinung an anderen Epithelien beobachtet, so zeigt sich einige Stunden post coitum in der Uteruswand der Maus und ganz besonders in der Schleimhaut eine : usgedehnte Leukozytose. Ganze Gruppen und Haufen von Leukozyten durchwandern jetzt das Zylinderepithel des Uterus. Auf cliese Weise gelangen immer 
mehr Leukozyten in die Lichtung des Organs und damit in das Ejakulat bzw. seine Reste. Schon bei relativ schwacher Vergrößerung (Fig. 1 Taf. XIV) ergeben sich auffällige Bilder.

Zeitlich fällt also die gleich näher $z u$ beschreibende intensive Leukozytose absolut genau mit dem Beginn der Auflösung des Ejakulates zusammen; sie setzt ein, wenn die Spermatozoen im Uterus absterben und zwar ganz plötzlich und rapid und ebenso schnell verschwindet sie wieder. Sie ist daher nur eine kurze Zeit lang zu beobachten, nur wenige Stunden hindurch; ihren Höhepunkt dürfte sie bei der Maus etwa 10-12 Stunden nach der Begattung erreichen. Untersucht man nicht gerade in der fraglichen Zeit von etwa nur 5 Stunden Dauer den Uterus, so hat man absolut keine Gelegenheit, diese auffällige und wichtige Erscheinung zu sehen.

Fast die gleichen Beobachtungen konnte $\mathrm{Kön}$ ig s t e i $\mathrm{n}$ bei der Ratte machen, daneben fand er den gleichen Vorgang der Leukozytose auch bei anderen Säugetieren, wie bei der Maus. Sonst liegen meines Wissens in dieser Hinsicht keine weiteren Befunde vor. Es erklärt sich das wohl dadurch, daß die Eier, die den Morphologen natürlich in erster Linie angehen, um diese Zeit noch im Eileiter liegen, so daß die gleichzeitige Untersuchung des Uterus meist unterblieb. So stützen sich die folgenden Mitteilungen auch fast ausschlieBlich auf Beobachtungen bei der Maus; sie werden

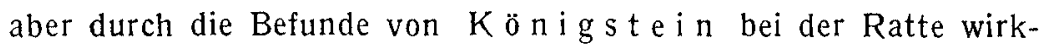
sam ergänzt.

Kö n i gste in (2) beschreibt nämlich das Verhalten des Uterus der Ratte nach der Begattung. Die Darstellung deckt sich fast vollkommen mit der, welche ich vor fast 25 Jahren von der Maus gegeben hatte (6). Das Sperma erfüllt beide Uterushörner derart, daß diese enorm stark ausgedehnt werden (von Streichholzzu Bleistiftdicke). Das Ejakulat besteht fast ausschließlich aus Spermatozoen, die 12-13 Stunden beweglich bleiben. Nach Erweichung des die Vagina ausfüllenden Pfropfes wird der größte Teil des Spermas in die Vagina entleert. Gleichzeitig treten zahlreiche Leukozyten in der Uteruslichtung auf, die die Uteruswand durchwandert haben, und nun den Rest der Spermatozoen phagozytieren. Königstein nimmt an, daß die Leukozystenemigration nicht auf den Reiz des in der Uteruslichtung gelegenen Ejakulates hin erfolgt, weil sie erst spät einsetzt. Dabei ist zber zu bederiken, daß Kö $\mathrm{nigst}$ e in den Begattungsakt selbst nicht 
beobachtet hat und anscheinend annimmt, daß die Begattung bei der Ratte unmittelbar nach dem Wurfe erfolgt. Oft vergehen aber nach meinen Erfahrungen eine Reihe von Stunden, bis der Koitus erfolgt. Es halten sich die Spermatozoen im Uterus der Ratte auch nicht so lange lebend wie $K o ̈ n i g s t e i n$ annimmt, son. dern nur etwa 5-6, höchstens wohl 10 Stunden, so daB man recht wohl daran denken könnte, daß das Ejakulat als solches den Reiz ausübt, daß aber einige Zeit vergeht, bis das Resultat aes Reizes morphologisch sichtbar wird.

Ich gehe nun zunächst zur Beschreibung der Verhältnisse bei der Maus über. Im nichtbrünstigen Zustand, aber auch bis zur Zeit der Begattung, ist die Tunica propria der Uterusschleimhaut der Maus nicht reicher an farblosen Blutzellen als andere Schleimhäute, $z$. B. die der Nase. Dieses ändert sich nun mit einem Schlage, wenn das Ejakulat einige Stunden die Uteruslichtung erfüllt hat; es treten jetzt massenhaft Leukozyten in der Tunica propria und auch im Epithel auf, und, wie gesagt, man wird wohl kaum fehl gehen, wenn man annimmt, daß diese Erscheinung die Folge eines auf die Uterusschleimhaut ausgeübten Reizes ist.

Diese Leukozytose der Uteruswand der Maus beginnt, wenn die Spermatozoen anfangen abzusterben; dann treten, wie ich früher schon beschrieb, die ersten Leukozyten im Ejakulat auf (7). Meine damals gegebenen Abbildungen lassen deutlich erkennen, daß das die Uteruswände um diese Zeit noch mehr oder weniger stark dehnende Ejakulat von Leukozyten zunächst noch völlig frei ist. Dieses besteht. vielmehr anfangs nur aus Spermatozoen und einzelnen Klümpchen einer amorphen azidophilen Masse, wahrscheinlich Samenblasensekret. Leukozyten treten im Ejakulat erst auf, wenn die Spermatozoen bewegungslos geworden sind. Dabei verlieren ihre Köpfe, wie ich schon früher beschrieb $(6,7)$, die so intensive Färbbarkeit mit basischen Farbstoffen. Bald sieht man nur noch vereinzelte, welche die normale Tinktion erkennen lassen, während die große Mehrzahl nur noch blaB oder teilweise (z. B. mit Hämalaun) gefärbte Köpfe erkennen läßt. Schließlich blaßt die Färbung immer mehr ab und die Spermaköpfe nehmen nur noch eine leichte Färbung mit sauren Anilinfarbstoffen (Eosin, Bordeaux) an. Die Schwanzfäden, die - solange die Spermatozoen lebend waren glatt und gestreckt im fixierten Präparat erschienen, sind jetzt unregelmäBig gekrümmt oder leicht aufgerollt. 
Fast das gleiche Verhalten der absterbenden Spermatozoen - aber der des Menschen in der Samenblase - beschreibt $K$ ö $\mathrm{n}$ i gst e in (2).

Wenn die Spermatozoen der Maus die beschriebene Veränderung durchmachen, die, wie erwähnt, mit der Zeit des Absterbens (Bewegungsloswerdens) zusammenfällt, beobachtet man auch die ersten Leukozyten in Ejakulat; zunächst noch verhältnismäßig wenige und nur in den Randgebieten des Ergusses. Alle gehören sie dem stark polymorphkernigen Typ an, den sie schon im Stadium der Durchwanderung des Epithels erkennen lieBen.

$\mathrm{Da}$ die Zahl der aus der Uteruswand auswandernden Leukozyten jetzt noch gering ist, ist auch das Bild, das die letztere bietet, noch kein so auffälliges wie wenige Stunden später. Mit der Zunahme der Zahl der Leukozyten im Ejakulat wächst auch die derer, welche die Uteruswand durchsetzen. Nicht blob die Tunica propria der Uterusschleimhaut enthält jetzt zahlreiche dichtgedrängte, farblose Blutzellen, sondern dieses gilt besonders auch für das Epithel, das nun streckenweise lebhaft an das der tonsillären Bildungen des Darmkanals erinnert; denn die durchwandernden Leukozyten liegen oft in Gruppen beisammen und bilden förmliche Nester im Epithel, ein für das Uterusepithel doch sehr auffälliges Bild. Ich habe es in Fig. 1 und 2 Tafel XIV dargestellt.

Wie, gesagt, sind alle diese durchwandernden Leukozyten polymorphkernig; sie enthalten drei bis fünf (oft auch mehr) anscheinend vollkommen getrennte, absolut pyknotische Kernpartikelchen, die in Halbring-, Ring- oder ähnlicher Form angeordnet sind. Nun vergleiche man damit die allerdings sehr primitiven Abbildungen von $\mathrm{Kohlbrugge}(3,5)$. Ich will zunächst die Angaben der ersten Mitteilungen von Kohlbrugge besprechen, die vor der meinigen erschienen sind und die Veranlassung zu meiner Stellungnahme gaben (3).

Ich glaube, daß jeder unparteiische Betrachter der beiden Abbildungen a und $\mathrm{b}$ von Kohlbrugge (3) die im Epithel liegend dargestellten Bildungen, welche Kohlbrugge S. 363 (3) beschreibt - ich komme auf die Beschreibung gleich zurück -, ohne weiteres für durchwandernde Leukozyten halten wird, und zu dieser Auffassung kam auch ich bei der ersten Lektüre der Mitteilung von Kohlbrugge. 
Meiner Ansicht nach verkennt Koh Ibrugge diese Bildungen eben völlig und das war der erste Schritt zu dem Irrtum, der seine ganze weitere Darstellung durchzieht. Die Worte, mit denen Ko h I b r u g e seinen Befund schildert, sind für die ganze Argumentierung bei der Deutung seiner Befunde so kennzeichnend, $\mathrm{daB}$ ich sie hier im Wortlaut wiedergeben muB, denn ich glaube nicht fehlzugehen, wenn ich den Kardinalfehler $\mathrm{Kohlbrugges}$ damit festlege.

Kohlbrugge schreibt (3) S. 363: ,Es ist mir aber aufgefallen, daß solche von Spermatozoiden durchzogene Mukosateile an einigen Stellen eigentümliche Kerne zeigen. Alle Kerne der Mukosa zeigen ein feines Fasernetz, in welchem dunkel gefärbte Körner liegen, von denen einige meist die anderen an Größe übertreffen.

Nun sieht man andere Kerne, in denen das Netzwerk verschwunden $z u$ sein scheint, das Plasma ist ganz hell und in der Mitte findet man nur ein großes Kernkörperchen, das wieder aus vielen dunklen Körnern zusammengesetzt ist. Auf Fig. a links ist solch ein Kern abgebildet. Noch eigenartiger ist der auf Fig. b abgebildete Kern mit stabförmigen Auswüchsen versehenen Kernkörperchen. Wiederum andere helle Kerne zeigen vier und mehr đunkel gefärbte Kugeln. Ob diese Form aus den beiden erstgenannten hervorgeht, läßt sich nicht feststellen, beachtenswert ist aber, daß die Spermatozoidenköpfe häufig einen hellen Hof zeigen, wie Fig. a beiderseits angegeben wurde; er fehlte den Spermatozoenköpfen der Fig. b. Manche Spermatozoidenköpfe sehen im Gewebe dicker aus als im Lumen, vielleicht schwellen sie alle an und gehen aus ihthen die hellen Kerne mit dunklen Kernkörperkugeln (Nukleolen?) hervor. An Zerfallsprodukte der Spermatozoiden darf man wohl nicht denken, denn dann würde die Masse nicht zunehmen, was hier zweifelsohne der.Fall ist, viel lieber nehme ich an, daß die Spermatozoiden sich mit den Kernen des mütterlichen Körpers vereinigen, daß aus dieser Vereinigung die hellen Kerne sich bilden, daß hier also ein Teilungsprozeß vorliegt."

Ich füge hinzu, daß aus Beschreibung und Abbildung von Kohlbrugge hervorgeht, daB er mit „Mukosa" und ,Mukosateilen" allein das Epithel meint. Die histologischen Unklarheiten, die mangelhafte Unterscheidung von Zelleib, Kern und Kernkörperchen in der Darstellung von $\mathrm{Kohlbrugge}$ faller dem Leser 
wohl ohne weiteres auf. Die Kerne mit dem Fasernetz, von denen Koh lbrugg e spricht, sind die Epithelkerne mit ihrem Chromatingerüst und den Nukleolen. Die Kerne, in denen , das Netzwerk verschwunden zu sein scheint", sind von Kohlbrugge verkannte durchwandernde Leukozyten, worüber Abbildung und Beschreibung kaum einen Zweifel lassen. Zum mindesten drei der von Kohlbrugge abgebildeten „Kerne" sind deutlich polymorphkernige Leukozyten.

Nun bildet $\mathrm{Koh} 1 \mathrm{~b}$ r u g g e aber noch andere, spermatozoenähnlichere Gebilde im Epithel $a b$, d. h. helle Höfe mit dunklem, länglich gefärbtem Inhalt, der entfernt an einen aufgequollenen Spermakopf erinnert. Diese Bilder haben Kohlbrugge wohl auf seinen grundlegenden Irrtum gebracht. Natürlich läßt sich aus den noch dazu sehr mangelhaften Abbildungen von $\mathrm{Koh}$ lbrugge kein sicherer Schluß ziehen, und ich kann daher das Folgende auch nur als Vermutung aussprechen, nämlich daB es sich auch hierbei um (stark geschrumpfte) Leukozyten handelt. Kohlbrugge hält dagegen die fraglichen Bildungen für gequollene Spermaköpfe, was ich natürlich von vornherein nicht bestreiten möchte (s. u.).

Kohlbrugge hat sein Untersuchungsmaterial sehr unzweckmäßig fixiert, nämlich mit Formol oder Pikrinschwefelsäure; namentlich die letztere Lösung ist ganz und gar ungeeignet, Organe von Säugetieren in gutem Erhaltungszustand $z u$ konservieren. Kleine $n b$ erg hat seinerzeit, als man über bessere Mittel noch nicht verfügte, dieses Gemisch für die Konservierung wirbelloser Tiere empfohlen; für Wirbeltiere und namentlich Säugetiere mit ihrem starken Gehalt an Bindegewebe ist die Pikrinschwefelsäure einfach unbrauchbar. Kohlbrugg e scheint selbst empfunden zu haben, daß seine Präparate nicht genügend fixiert waren; sagt er doch selbst (1. c. S. 363): ,Mein Material genügt nicht, um in dieser Frage Klarheit zu erlangen; cas muß späteren Forschungen an vielleicht in anderer und besstrer Weise gehärtetem Material überlassen bleiben."

Ich bin nun in meiner längst gefaßten, aber vorsichtshalber bisher noch nicht geäußerten Ansicht, daß die Veränderungen, die Kohlbrugge an Spermatozoen beschreibt, die in die Uteruswand der Fledermaus eindringen, auf Verwechslung mit emigrierenden Leukozyten beruhen, durch zwei Aeußerungen von Kohl- 
brugge selbst bestärkt wurde. Erstlich sagt Kohlbrugge in seiner Nachschrift, die er seiner ersten Veröffentlichung (i. c S. 368) anfügt: ,Nach AbschluB dieser Arbeit stieB ich noch auf einen Uterus, in dem die Spermatozoiden noch die volle Aktivität des Eindringens zeigen. In den Schnitten sieht man eine ungeheure Menge Kerne, welche die vier S. 363 genannten Kugeln zeigen, in anderen haben sich diese Kugeln ausgedehnt, und man sieht nun vier Kerne, die dann an anderen Stellen auseinanderweichen." Ich deute mir diese Angabe von Kohlbrugge so, daß er nun ein Präparat in die Hand bekam, das auf der Höhe der Leukozytenemigration stand; denn daß die „,Kerne mit den vier Kugeln“ polymorphkernige emigrierende Leukozyten sind, unterliegt mir insbesondere mit Rücksicht auf das im folgenden Auszuführende keinem Zweifel mehr.

Ferner hat sich dann $\mathrm{Ko}$ h l b r ugge gleichzeitig mit meiner Stellungnahme gegen ihn (7) und infolgedessen ohne Kenntnis meiner Mitteilung erneut mit dieser Frage befaßt (5). Da ich diese Veröffentlichung von Kohlbrugge bei meiner ersten Erwiderung noch nicht kennen konnte, habe ich noch nicht Gelegenheit gehabt, auf sie einzugehen. Kohlbrugge legt sich hier (5) nochmals die Frage vor: Was wird nun bei Säugetieren aus den in die Mukosa eingetretenen Spermatozoiden? und er antwortet jetzt wesentlich vorsichtiger: ,Mein Material genügt nicht, um in dieser Frage Klarheit zu erlangen."

Im wesentlichen handelt es sich um die Beschreibung der stark mit Spermatozoen angefüllten Drüsenschläuche der von Koh 1brugge untersuchten Fledermausart. Die histologische Beschreibung zeigt die gleichen Unklarheiten wie früher (3) und neben der Verkennung der emigrierenden Leukozyten hat es den Anschein, als laufe $\mathrm{Kohlbrugge}$ noch eine andere Verwechslung unter. Ko h lbrugg e (5) schreibt nämlich: ,1. Das Epithel der Drüsenschläuche zeigt in der Regel helle Zellen mit hellen großen Kernen (Fig. 1 usw.); sehr oft werden diese hellen Zellen aber wie auf Fig. 1 von dunkelpigmentierten, stabförmigen Gebilden unterbrochen. Sie zeigen sehr verschiedene Formen, zuweilen laufen sie keilförmig spitz zu, zuweilen sind sie mehr oval, seltener haben sie sich zur Breite der hellen Mukosakerne ausgedehnt."

Es geht zunächst daraus also wieder hervor, daB $\mathrm{Kohl-}$ hrugge mit ,Mukosakernen“ Epithelkerne meint, was auch 
für den weiteren Verlauf seiner Darstellung der Fall ist. Ko h lb rugge hat nun bei der von ihm untersuchten Fledermausart gesehen, daß Spermatozoen reihenförmig gegen die Uteruswand gestellt sind, wie das neuerdings auch G ro Be r (1) für den Maulwurf kurz beschreibt und abbildet. Von diesen Spermatozoen hat $\mathrm{Koh} \mathrm{l} \mathrm{b} \mathrm{r} \mathrm{g} g$ e (ebenfalls in Uebereinstimmung mit den Angaben von Grosser, die aber keineswegs verallgemeinert werden dürfen) eine Anzahl in das Epithel eindringen sehen. Durch schlecht erhaltene Präparate hat sich Kohlbrugge verleiten lassen, anzunehmen, daß die Köpfe dieser Spermatozoen in der Uteruswand eigenartige Veränderungen eingehen (s. oben). Nun hält Kohlbrugge alles, was einigermaßen spermatozoenähnlich aussieht, für Umbildungsprodukte von Samenfäden. Während er bisher und - wie ich nun glaube mit absoluter Sicherheit nachweisen $z \mathfrak{u}$ können - auch im folgenden einer Verwechslung mit emigrierenden Leukozyten zum Opfer gefallen ist, verkennt er jetzt daneben noch eine andere Bildung. Soweit das aus den keineswegs klaren Abbildungen von $\mathrm{Kohlbrugge}$ zu erkennen ist, handelt es sich bei den schmalen, ,dunkelpigmentierten“ (!) Zellen um jene in den meisten Zylinderepithelien regelmäßig vorkommenden Kompressionsformen von Epithelzellen, die meist als Stiftchenzellen benannt werden. Sie sind von verschiedenen Autoren auch für das Uterusepithel der Säugetiere beschrieben worden. Natürlich verkennt Kohlbrugge, wenn meine Deutung richtig ist, auch die Chronologie des Vorganges.

Viel wichtiger für die Klärung der zwischen $\mathrm{Kohlbrugge}$ und mir bestehenden Differenzen ist aber die folgende Darstellung von Kohl brugge. Er lehnt sich wieder an seine erste Mitteilung an, indem er schreibt: ,2. Die großen hellen Kerne der Mukosa zeigen in der Regel ein feines Netzwerk, in welchem dunkel gefärbte Körner liegen, von denen einige wenige immer die Mehrzahl an Größe übertreffen. Nun sieht man häufig zwischen diesen gleichmäßig gebildeten Kernen andere auftreten, in denen alle Körner sich in der Mitte zu einem dunklen, großen, runden Kernkörperchen zusammengezogen haben, das von einem hellen Hof umgeben ist. Einige dieser Kernkörperchen zeigen Verzweigungen und erinnern dadurch an die Chromosomenknäuel sich teilender Zellen. 3. In anderen solchen wasserhellen Kernen ist das Kernkörperchen in mehrere Kugeln auseinandergefallen; man sieht sie 
sowohl im Drüsenepithel als auch im interstitiellen Gewebe. Auffallend ist, daB auch die Spermatozoen, wenn sie in das Gewebe eingedrungen sind, wobei sie stets den Schwanz verlieren, von einem hellen Hof umgeben sein können, der an Enzymwirkung erinnert. Dadurch werden diese Spermatozoenköpfe obengenannten Kernen ähnlich. 4. An anderen Stellen, und zwar im interstitiellen Gewebe, sieht man eine große Anhäufung kleiner dunkler Kerne, die auch aus Spermatozoiden entstanden aufgefaßt werden können. 5. Manche Durchschnitte scheinen zu der Auffassung zu berechtigen, daß die Spermien als Ganzes (ohne den Schwanz) in die großen Mukosakerne eindringen und sich dort direkt in die dunklen Körner dieser Zellen umwandeln."

Es handelt sich also bẹ̣ dieser Beschreibung um ähnliche histologische Unklarheiten wie in der ersten Veröffentlichung von $\mathrm{Kohl-}$ brugge (3). Kern, Kernkörperchen usw. werden wahllos durcheinander verwendet und sicher für ganz verschiedene Dinge. Die Nukleolen der Epithelkerne werden für Spermienköpfe (? klar ist das, was Kohlbrugge meint, überhaupt nicht) gehalten, emigrierende Leukozyten (als solche auf den Figuren von $\mathrm{Ko}$ h lbrugge deutlich erkennbar) für umgewandelte Spermienköpfe oder Verschmelzungsprodukte (?) solcher mit Epithelkernen. Leukozyten der Tunica propria (Fig. 3), die trotz der Unvollkommenheit der Abbildungen von $\mathrm{Koh} / \mathrm{b} \mathrm{r}$ ug e unverkennbar sind, werden wiederum als Umwandlungsprodukte von Spermatozoen erklärt. Die Tunica propria der Schleimhaut nennt Kohlbrugge ïbrigens recht ungewöhnlichẹweise ,interstitielles Gewebe“.

Nun macht aber Kohlbrugge (5) bei seinen Erklärungsversuchen der histologischen Befunde trotz der vorgefaßten Meinung, daß Spermatozoen in die Schleimhaut eintreten, ein wichtiges Geständnis. Er schreibt nämlich: ,Noch schwieriger wird die Sache dadurch, daß ein Uterus, in dem keine Spermatozoiden nachzuweisen sind, auch bei ganz jugendlichen Tieren dieselben Formen zeigen können,-die ich oben unter $1-4$ beschrieben habe. Da könnte man also schließen, daß sie alle nichts mit den Spermatozoiden zu tun haben."

Diesen Schluß hätte Kohlbrugge nur ziehen sollen; es ist mir sogar ganz unbegreiflich, daß er trotz des Befundes, daB die von ihm so arg mißverkannten histologischen Bilder auch ohne Spermatozoen auftreten können, nicht in sich gegangen ist und 
sich gesagt hat, also habe ich mich geirrt; was ich für eingedrungene und umgewandelte Spermatozoen gehalten habe, können solche nicht sein, denn ich finde die gleichen Bilder auch dann, wenn gar keine Spermatozoen da sind oder da waren. Ich erwähnte ja schon oben, daß auch im nichtbrünstigen Zustand des Uterus eine gewisse Leukozytenemigration stattfindet, wie in allen Schleimhäuten. DaB diese Erscheinung sich aber enorm steigert, wenn das Ejakulat resorbiert wird, wurde oben ebenfalls erwähnt.

Daher auch die etwas seltsam anmutende Entschuldigung seiner Auffassung seitens Kohlbrugges, daß der Vorgang, den er für Umwandlungserscheinungen von Spermatozoen in der Uterusschleimhaut hält, zwar bei jugendlichen Tieren und ohne Anwesenheit von Spermatozoen bereits vorkommt, daß dieser aber zur Zeit nach der Begattung sich enorm steigert. Sehr richtig! Dann wird eben der sonst nur geringgradige Vorgang der Leukozytenemigration ein enorm starker.

Ith bin mir sehr wohl bewußt, daß ich hier gegen einen Forscher einen recht schweren Vorwurf erhebe, nämlich den, daß er auf Grund schlecht erhaltener-Präparate Behauptungen aufgestellt hat, die den Tatsachen keineswegs entsprechen. Es mag im ersten Augenblick anmaßend erscheinen, daB ich es wage, die recht positiven, wenn auch in einigen Schlußfolgerungen etwas zögernd ausgesprochenen Behauptungen von Kohlbrugge als absolut verfehlt hinzustellen.

Ich habe bisher auch gezögert, das zu tun, obwohl ich, wie gesagt, von vornherein nicht im Zweifel darüber war, daß meine Vermutungen berechtigt seien. Und so schwer es mir fällt, meinen Standpunkt in der Frage so offen zu proklamieren, so sehe ich mich doch dazu genötigt, es hier zu tun, weil ich mehr als einmal die Beobachtung gemacht habe, daB von seiten anderer Fachgenossen wie Physiologen, Kliniker, derartige Mitteilungen aufgegriffen und als Tatsachen in die weitere medizinische Literatur übernommen werden.

Ich bin also der Ansicht, daB die sonst nirgends bestätigte Anschauung von Kohlbrugge, der aber eine große Anzahl sicherlich weit besser ausgeführter positiver Beobachtungen gegenüberstehen, daß die überzähligen zur Befruchtung nicht Verwendung findenden Spermatozoen in die Uteruswand der Säugetiere eindringen und dort mit den Gewebskernen (Epithel) in Beziehung 
treten, wenigstens was den letzten Punkt anlangt, irrig ist. Ich spreche die Vermutung aus, daB sich Kohlbrugge durch die im Uterusepithel befindlichen, auf der Auswanderung ins Uteruslumen begriffenen Leukozyten hat täuschen lassen, wozu der mangelhafte Erhaltungszustand seiner Präparate wesentlich beitrug. Kohlbrugge hat wahrscheinlich die teilweise woh! stark geschrumpften Kerne der emigrierenden Leukozyten mit Spermaköpfen verwechselt, wie aus seinen Beschreibungen und Abbildungen hervorgeht.

Vor allem aber ist der Irrtum, dem Kohlbrugge verfallen ist, daraus deutlich zu folgern, daB er selbst genötigt ist, anzugeben, daB die von ihm als Umwandlungsprodukte von Spermatozoen aufgefaßten Bildungen auch dann in die Erscheinung treten, wenn gar keine Spermatozoen da sind. Damit ist also einfach ausgeschlossen, daß es sich um das handelt, was Koh ibrugge annimmit.

Ko h lbrugge (3-5) hat anscheinend das Gebiet, mit dem er sich in seinen Veröffentlichungen beschäftigt hat, nicht genügend beherrscht; darauf deuten auch seine vielfach ganz unklaren allgemein-histologischen Vorstellungen hin, die er mehrfach entwickelt. Ebensowenig präzise waren die Auffassungen der in Frage kommenden Entwicklungsvorgänge, die ich zusammen mit der mangelhaften Berücksichtigung der Literatur schon früher (7) beanstandete.

Das beste wäre, Herr $\mathrm{Koh} 1 \mathrm{brugge}$ legte seine diesbezüglichen Präparate auf einer Anatomenversammlung einem Kreise von Fachgenossen zur Begutachtung vor.

Ich komme nun nach dieser erneuten Polemik gegen $\mathrm{Kohl-}$ brugge auf das eigentliche Thema meiner Mitteilung zurück, auf das Schicksal der Spermatozoen im Uterus. Einerseits sterben also die bewegungslos gewordenen Spermatozoen ab, wobei sie charakteristische Veränderungen erkennen lassen (s. oben), andererseits wandern immer mehr Leukozyten ins Uteruslumen ein.

Kö n ig stein (2), der diese Erscheinung zuerst richtig beschrieben hat, nimmt auf Grund seiner Befunde an, daß die Leukozyten die Aufgabe haben, die im Uterus zurückgebliebenen Spermatozoen durch Phagozytose zu vernichten. Es unterliegt keinem Zweifel, daB gelegentlich auch bei der Maus Spermatozoen phagozytiert werden. Man findet namentlich bei Beginn der Leukozyteneinwanderung in das Ejakulat bzw. seine Reste in dessen 
Was wird aus den in den Uterus ejaculierten usw. Spermatozoen? 201

Randpartien Leukozyten, die Spermaköpfe in ihrem Plasma enthalten, und zwar kann man an diesen Spermaköpfen dieselben Veränderungen erkennen, die sich an den frei im Ejakulat liegenden vollziehen, d. h. die Färbbarkeit des Chromatins nimmt allmählich $a b$, ehe die volle Auflösung des phagozytierten Kopfes erfolgt ist. Auch sonst - selbst in späteren Stadien der Auflösung des Ejakulats - kann man hin und wieder diese Beobachtung machen.

Aber die Erscheinung ist doch zu selten und die Zahl der Leukozyten, die Spermaköpfe phagozytieren, steht in gar keinem Verhältnis zur Menge der Spermatozoen, ferner kommt auch das Gros der Leukozyten für diesen Zweck zu spät, da die bei weitem größte Masse der Spermatozoen schon in der oben beschriebenen Weise ohne nachweisliche Tätigkeit der Leukozyten im Sinne einer Phagozytose zugrunde geht.

Selbst wenn man annimmt, daß viele der Leukozyten, die man ohne in Phagozytose begriffenen Spermaresten antrifft, bereits einen oder mehrere Spermatozoen verdaut haben, besteht noch immer ein außerordentliches Mißverhältnis zwischen beiden Elementen. Dazu kommt, daß die Leukozyten, wie gesagt, eigentlich zu spät kommen, um noch nennenswert durch Phagozytose wirken zu können. So scheint mir doch trotz der großen Verlockung, den Leukozyten in erster Linie die Rolle der Phagozytose zuzuschreiben, das nicht angängig $z u$ sein. Im Zentrum des Ejakulates beobachtet man schon einen hochgradigen Zerfall der Spermatozoen, wenn der Vaginalpfropf noch hält und das Ejakulat noch nicht nennenswert an Größe eingebüßt hat. Dann fehlen die Leukozyten noch fast ganz oder treten erst ganz vereinzelt in den Randpartien auf.

Aber auch die Lebensdauer der ins Uteruslumen eingewanderten Leukozyten ist eine beschränkte. Sie gehen mit den Resten des Ejakulates zusammen zugrunde. Man beobachtet nämlich folgendes: Sehr bald nachdem die Leukozyten ins Ejakulat eingedrungen sind, werden ihre Kerne noch stärker polymorph und die einzelnen Kernpartikelchen werden vollkommen pyknotisch. Die zellige Abgrenzung der Leukozyten gegenüber den umliegenden Resten des Ejakulats wird undeutlich, schließlich löst sich das Protoplasma volikommen auf und man beobachtet nur noch die Kernreste. Dieser Vorgang wird um so deutlicher, je weiter die Leukozyten in das Ejakulat vordringen, je länger sie also auch die Uteruswand verlassen haben (Fig. 3 Taf. XIV). 
Sie verfallen hier eben der völligen Auflösung, die sich schließlich darin bemerkbar macht, daß man einen Haufen feinster chromatischer punktförmig erscheinender Kügelchen an Stelle der ehemaligen Leukozyten beobachtet. Es ist also zu einer Chromatolyse des Kerns gekommen.

Man könnte im ersten Augenblick beim Betrachten dieser chromatischen Punkthäufchen schwanken, ob man es mit Zerfallsprodukten der Leukozyten oder der Spermatozoen zu tun hat. Wenn man aber bedenkt, wie oben bereits ausgeführt, daß die Spermaköpfe sehr bald nach dem Absterben ihre Färbbarkeit einbüßen, die Kerne der Leukozyten aber immer stärker pyknotisch und daher intensiver färbbar werden, daß ferner die ungefähr kuglige Form, in der die Körnchenhaufen sich finden, der Gestalt der Leukozytenkerne annähernd entspricht, so kann kein Zweifel bestehen, daß man es mit den Resten der Leukozyten zu tun hat.

So verlockend es also auch ist, anzunehmen, wie das $\mathrm{K} \ddot{0} \mathrm{n} \mathbf{i g -}$ s te i n tut, daß die starke Leukozytenemigration lediglich dazu dienen soll, die Spermatozoen zu phagozytieren, die Tatsachen sprechen nicht dafür. Es scheint fast, als ob nur die Spermatozoen phagozytiert würden, die noch beweglich waren und aktiv in die Leukozyten eingedrungen sind, daher die geringe Zahl der farblosen Blutzellen, die Spermatozoenköpfe enthalten. Die Frage nach der Funktion der Leukozyten muß also zunächst offen bleiben. Da positive Unterlagen zunächst nicht vorliegen, enthalte ich mich jeder Hypothese. $Z \mathbf{u}$ denken gibt aber vielleicht die Tatsache, daß ihre Lebensdauer nach dem Eintritt in das Ejakulat eine so geringe ist. Vielleicht sollen sie die Uteruswand vor Invasion durch Spermatozoen schützen?.Vielleicht stellen die von $\mathrm{Kohl}$ b r $\mathrm{g} g \mathrm{ge}$ 3,5) dargestellten Bilder emigrierende Leukozyten dar, die eindringende Spermatozoen phagozytieren?

Mittlerweile ist der Vaginalpfropf der Maus so weit erweicht, daß er ausgestoßen werden kann. Damit kann der zu Detritus verwandelte Rest des Ejakulates samt den in ihm zugrunde gegangenen Leukozyten aus den weiblichen Genitalwegen abfließen, so daß, wenn nach vollendeter Furchung die Eier die Uteruslichtung betreten, diese bereits völlig frei von Resten des Ejakulates ist und sich nicht mehr von dem Zustand vor der Begattung unterscheidet. 
Diese Angaben beziehen sich natürlich nur auf das Verhalten des Ejakulates bei der Maus. Ganz gleich dürften die Erscheinungen bei der Ratte sein, wie ja aus der Darstellung von $K$ ö $n$ i g s t e i n (2) hervorgeht. Leider habe ich weder beim Meerschweinchen noch heim Kaninchen den Uterus in den fraglichen Perioden beachtet, so $\mathrm{daB}$ ich von diesen Tieren keine positiven Angaben machen kann. Ich zweifle aber nicht daran, daß trotz mancher Verschiedenheiten im einzelnen, die sicherlich vorhanden sein werden, doch die grundlegenden Verhältnisse die gleichen sind. Natürlich wäre es sehr empfehlenswert, wenn diese Fragen auch hier an einem einwandfreien Material erneut untersucht werden könnten.

Ich gebe nun im Anschluß an diese Beschreibung einige Abbildungen auf Taf. XIV. Fig. 1 zeigt bei 300 facher Vergrößerung einen Teil der Uteruswand der Maus zur Zeit der Leukozytendurchwanderung; man überblickt die ganze Dicke der Schleimhaut bis an die Grenze der Muskelhaut.

In der Tunica propria trifft man neben den fixen Zellen zahlreiche Leukozyten, von denen die Mehrzahl zwar schon polymorphkernig ist, eine größere Anzahi aber noch kompaktere Kernformen erkennen läßt, namentlich Ringkerne. Wenn die Leukozyten das Epithel erreicht haben, sind sie fast ausnahmslos polymorphkernig. Oft sind es ganze Gruppen von Leukozyten, die im Epithel getroffen werden (in Fig. 1 rechts).

Bei dieser Gelegenheit möchte ich die Frage kurz streifen, woher die durchwandernden Leukozyten stammen. Man wird in erster Linie annehmen, daß sie aus dem strömenden Blute kommen. In der Tat zeigt Fig. 1 in der Nähe des BlutgefäBes links oben eine Gruppe von Leukozyten, von denen man vermuten könnte, dab sie die Gefäßwand eben durchsetzt haben.

Wenn man aber bedenkt, daß zum mindesten eine große Zahl von Leukozyten der Tunica propria Kernformen zeigt, die im strömenden Blute selten sind, und wenn andererseits Uebergangsformen zwischen den kleinen rundkernigen Zellen der Tunica propria und den noch nicht polymorphkernigen Leukozyten nicht zu verkennen sind, so wird man die Möglichkeit, wenn nicht Wahrscheinlichkeit, offen halten müssen, daß die genannten Propriazellen emigrierende Leukozyten liefern können.

Es lassen sich nämlich (außer Leukozyten) im wesentlichen drei Zeliformen in der Propria der Uterusschleimhaut der Maus 
unterscheiden; erstlich längliche Elemente mit länglichen, ziemlich chromatinarmen Kernen, die sich deutlich als gewöhnliche Fibroblasten $\mathrm{zu}$ erkennen geben; zweitens viel zahlreichere Rundzellen mit runden, wesentlich chromatinreicheren Kernen, die anscheinend in Leukozyten unmittelbar übergehen können; drittens größere Zellen mit größeren, etwas unregelmäßig geformten Kernen, die besonders stark chromatinreich sind. Die zweite und dritte Zellform dürfte in naher Verwandtschaft zu der großen Gruppe der farblosen Blutzellen stehen.

Fig. 2 zeigt dann bei wesentlich stärkerer Vergrößerung die Durchwanderung des Epithels seitens der Leukozyten. Besonders stark ist hier eine Ansammlung der Leukozyten dicht unter dem Epithel erkennbar.

In Fig. 3 ist ein Ausschnitt aus dem Ejakulatreste dargestellt, nachdem die Spermatozoen abgestorben sind und die Leukozyten ebenfalls bereits anfangen zu zerfallen. Ein Spermatozoon ist noch sichtbar, daneben zahlreiche, stark polymorphkernige Leukozyten und alle Stadien der Karyolyse ihrer Kerne, wobei die Zellform als solche schließlich ganz verschwindet und ein Detritus an deren Stelle tritt.

\section{Literaturverzeichnis.}

1. Grosser, Ot to, Die Aufgaben des Eileiters der Säugetiere. Anatem. Anzeig. Bd. 50. 1918.

2. Kön igste in, H., Ueber das Schicksal der Spermatozoen, welche nicht zur Befruchtung gelangen. Wiener klinische Wochenschr. Bd, 21. 1908.

3. Kohlbrugge, J. H. F., Der Einfluß der Spermatozoiden auf den Uterus. Zeitschr. für Morphologie und Anthropologie. Bd. 12. 1910.

4. De r s el be, Der Einfluß der Spermatozciden auf die Blastula. Archiv für mikroskopische Anatomie. Bd. 75. 1910.

5. Derselbe, Ueber den Einfluß der Spermatozoiden auf den Uterus. Zeitschr. für Morphologie und Anthropclogie. Bd. 13. 1911.

6. Sobotta, J., Die Befruchtung und Furchung des Eies der Maus. Ebenda. Bd. 38. 1895.

7. Derselbe, Ueber das Verhalten der Spermatozoen im Uterus der Säugetiere. Nach den Befunden bei Nagetieren (Maus, Ratte u. a.) Zeitschrift für Morphologie und Anthropologie. Bd. 13. 1911.

8. D e r s e 1 b e , Ueber den Mechanismus der Aufnahme der Eier der Säugetiere in den Eileiter und des Transportes durch diesen in den Uterus. Nach Untersuchungen bei Nagetieren (Maus, Ratte, Kaninchen, Meerschweinchen). Anatomische Hefte. H. 163 (Bd. 54). 1916. 


\section{Erklärung der Figuren auf Taf. XIV.}

Fig. 1. Teil der Uteruswand der Maus zur Zeit der Leukozy tose der Schleimhaut bei Resorption des Ejakulates. Verg. $300: 1$.

Fig. 2. Epithel und angrenzender Teil der Tunica propria der Uterusschleimhaut in der gleichen Zeitperiode. Vergr. $800: 1$.

Fig. 3. Teil des in Auflösung begriffenen Ejakulates im Uterus der Maus. Vergr. $700: 1$.

A n merkung 1. Die Tatsache, aut die A doI p h i (Anat. Anzeiger Bd. 26 und 27, 1905 und 1906) die Aufmerksamkeit gelenkt hat, nämlich đaß die Spermatozoen der Säugetiere gegen den Strom schwimmen können, wenn dieser nicht zu stark ist, wird in ihrer physiologischen Bedeutung gewöhnlich ïberschätzt, insofern als angenommen wird, daß der Flimmerstrom des Epithels der weiblichen Genitalwege das Aufsteigen der Samenfäden in diesen begünstige.

Für viele Säugetiere fällt diese Annahme schon dadurch, daB der Uterus (und bei manchen auch der Eileiter) überhaupt kein flimmerndes Epithel besitzt (s. u. Anmerkg. 2). Außerdem wird ja bei der Maus zum Beispiel das Sperma direkt in den Uterus ejakuliert und füllt diesen vollkommen an. Auch für das Eindringen in den Eileiter kann der Flimmerstrom nicht verantwortlich gemacht werden, weil die uterine Hälfte des Eileiters bei der Maus und auch anderen Säugetieren ebenfalls nicht flimmert oder die Auskleidung mit Flimmerepithel jedenfalls nicht konstant ist. Ferner sehen wir, daß auch da, wo die uterine Hälfte des Eileiterepithels flimmert, wie beim Kaninchen (vor der Befruchtung und kurze Zeit nachher) oder der Ratte ${ }^{1}$ ) die Spermatozoen nur in geringer meist sogar ganz außerordentlich geringer Zahl in den Eileiter eintreten. Ferner besteht zwischen Maus und Ratte trotz des eben erwähnten Unterschiedes der Flimmerung des Eileiters keine Verschiedenheit in der Zahl, in der die Spermatozoen in die Tube vordringen, und keine in der Zeit, in der das geschieht.

Anmerkung 2. Grosser (1) hat kürzlich die Frage, ob der Uterus der Säugetiere flimmert oder nicht, nochmals angeschnitten und in dankenswerter Weise eine sehr umfangreiche Literatur zusammengestellt, darunter auch solche, die mir bisher unbekannt war und auch leider unzugänglich ist. $\mathrm{DaB}$ im Vorkommen von Flimmerzellen im Epithel namentlich eines Organes wie des Uterus, der im jugendlichen Zustand auch dann Flimmerepithel trägt, wenn das später nicht mehr der Fall ist, große individuelle

$\left.{ }^{1}\right)$ Grosser (1) hat recht, wenn er meine letzten Angaben (8) bezüglich der Ratte korrigiert und zwar mit meinen eigenen Angaben. In der Tat tlimmert namentlich zur Zeit vor und nach der Begattung fast die ganze uterine Strecke der Tube, die bei der Maus nie Flimmerepithel trägt; erst zur $Z$ it der Furchung scheint sich die Mehrzahl der Flimmerzellen in stzernierenden Zellen umzubilden. 
und lokale Verschiedenheiten vorkommen mögen, ist begreiflich, wenn man bedenkt, daß Flimmerzellen und Inseln solcher gelegentlich sogar mitten in anderen Epithelien vorkommen wie selbst geschichtetem Pflasterepithel.

$\mathrm{Ob}$ aber Flimmerepithel vorhanden ist oder nicht, das zu entscheiden ist ja doch genügende Fixierung vorausgesetzt - für jeden einigermaßen erfahrenen Mikroskopiker auch am fixierten Präparat leicht möglich. Wenn Grosser (1) kürzlich schreibt, daß wenn andere Autoren das Flimmerepithel namentlich am frischen Präparat festgestellt haben, ,so kann man diese Angaben nicht einfach auf Grund fixierter Präparate wegleugnen", so bin ich mit dieser Anschauung ebenso wenig einverstanden, wie mit der Angabe ,zweifellos sind die Flimmerhaare sehr labil", wobei Grosser doch wohl meint, die Autoren, die ihre Anwesenheit leugnen, hatten Epithelien mit abgefallenen Flimmerhaaren vor sich.

Eine Flimmerzelle ist doch nicht eine einfache Zylinderzelle mit Flimmerhaaren an der Oberfläche, sondern der ganze Bau der Zelle wird durch die Flimmereinrichtung beeinflußt; und wenn die Flimmerhaare beschädigt sind, sieht man doch zum mindesten Reste von ihnen, jedenfalls aber die Basalkörperchen. Und wenn man lebenswarmes Material fixiert, so sollen auch Artefakte an den Flimmerhaaren selbst nicht vorkommen und man soll solche Angaben eben nur auf Grund der Befunde an lebenswarmem Material machen.

Wenn ich auf der gleichen Schnittserie wcmöglich sogar auf dem gleichen Schnitte nebeneinander das wunderbar schöne und durch einige von mir und anderen beschriebene Besonderheiten ausgezeichnete Flimmerepithel deś Infundibulum tubae und des ampullären Eileiterabschnittes der Maus neben dem ganz anders gestalteten nichtflimmernden Epithel des isthmischen Teils und reben $\mathrm{d} t \mathrm{~m}$ Epithel des Uterus sthe, so erlaube ich mir in der Tat, wenn ich bei vielen hundert Tieren stets das gleiche Resultat finde, ein positives Urteil zu fällen. Und wenn ein anderer zulfälliger Beobachter einmal ein Paar Flimmerzellen im Uterus findet, so ist das eben ein ganz unwesentlicher Befund: Und bei der nahe verwandten Ratte flimmert das Eileiterepithel zur Zeit der Befruchtung und auch einige Zeit nachher auch da, wo das bei der Maus nie der Fall ist und erstreckt sich in gleicher Eigenschaft anscheinend (genauere Angaben kann ich zur Zeit nicht machen) noch eine Strecke weit in den Uterus hinein.

Eine noch so beschädigte Flimmerzelle ist auch am (gutfixierten) Präparat stets noch zu erkennen. Und ich glaube, daß auch andere Autoren es sich verbitten werden, daß man ihre negativen Befunde etwa im Sinne von Kunstprodukten verwertet. Wie gesagt, kenne ich kaum ein schöneres Demonstrationsobjekt für eine Flimmerzelle als die gut erhaltene Zelle aus dem weiblichen Genitaltrakt der Nager, namentlich der Ratte und Maus. Die nicht flimmernden Zylinderepithelien dieses Traktus sind selbst von einem Anfänger mit den Flimmetzellen nicht zu verwechseln.

Und wenn bei ganz systematischen Untersuchungen auf diesen Gegenstand hin wie denen von B e il i n g (Arch. f. mikrosk. Anatcm. Bd. 67, 1906) und $\mathrm{Kell}$ e r (Anat. Hefte Bd. 39, 1909) z. B. beim Hunde kein Flimmerepithel im Uterus gefunden wird, so beweisen anders lautende Befunde nur, 
Was wird aus den in den Uterus ejaculierten usw. Spermatozoen? 207

daß hier individuelle und lokale Verschiedenheiten vorkommen neben den wohl noch nicht völlig geklärten rhythmischen Aenderungen des Epithelcharakters.

Was die positiven Angaben namentlich die der älteren Literatur anlangt, so bedenke man immerhin zweierlei: 1. die damals zur Verfügung stehenden Methoden waren noch keine ganz einwandfreien; wurden mit ihnen negative Befunde erzielt, so konnten diese nicht genügend' geschätzt werden; 2. positive Resultate ab€r, selbst wenn sie zufällige waren, wurden leicht verallgemeinert. So hatte sich die Anschauung weiteste Bahn gebrochen, daß der Uterus aller Säugetiere mit Flimmerepithel ausgekleidet sai. Unter diesem Wahne - denn daß diese Anschauung nicht richtig ist. wird wohl heute nicht mehr bestritten - stand auch anscheinend $K$ oh lb r u g g e $(3,5)$, wenn er die Beziehungen der Spermatozoen zur Uteruswand der von ihm untersuchten Fledermausart auf die Anziehung seitens des Flimmerstroms zurückführt. Kohlbrugges eigene, leider ja keineswegs glänzende Abbildungen lassen aber von Flimmerepithel nicht das Geringste erkennen.

Königsberg, im März 1919. 